\title{
Hemifacial motor and crying seizures of temporal lobe onset: case report and review of electro-clinical localisation
}

\author{
R E Hogan, V K Rao
}

J Neurol Neurosurg Psychiatry 2006;77:107-1 10. doi: 10.1136/jnnp.2005.062554

Objective: To report a case of temporal lobe epilepsy with clinical presentation of paroxysmal episodes of "tightness" over the right hemiface, and ictal crying, and review electroclinical localisation of this phenomenon.

Methods: Clinical semiology, neurophysiological localising tests, and epilepsy surgery outcome are reported in a subject presenting with paroxysmal right hemifacial movements and ictal crying. Pertinent past reports of somato-motor signs and ictal crying in temporal lobe epilepsy are reviewed and the findings correlated with proposed human facial cortical representation.

Results: Simple partial seizures caused by temporal lobe epilepsy presented with right sided tonic facial movements and ictal crying. Intracranial EEG monitoring documented a left medial temporal onset of seizures that remained asymptomatic until they propagated to the left cingulate region. Anterior temporal lobectomy with resection of the amygdala and anterior hippocampus resulted in cessation of seizures.

Conclusions: This is a rare example of epileptic seizures of medial temporal onset presenting with isolated somato-motor manifestations and ictal crying. Anatomical-electrical-clinical correlations with cortical regions controlling facial movements were highly suggestive that this case represents secondary activation of "emotional" motor cortex M3 and M4 (rostral and caudal cingulate motor cortex), giving rise to focal hemifacial movements and ictal crying.

\section{CASE REPORT}

A 28 year old right handed man had onset of epilepsy at eight years of age. Seizures were simple partial with right hemifacial tonic and tonic-clonic movements, with no associated symptoms in the extremities. Typical seizure duration was 10-30 seconds. Occasionally, more prolonged seizures involved "grunting" respirations, and some degree of loss of consciousness. There were no associated affective or "experiential"1 symptoms, such as fear or pain. Seizures were sometimes associated with lacrimation.

At presentation, his simple partial seizures were occurring three to four times a day. The seizures were refractory to multiple antiepileptic drugs. Neurological examination on presentation was normal except for postural tremor in both hands.

During non-invasive video EEG telemetry monitoring the patient had 12 seizures over a three day period. The typical ictal semiology was initial tonic contraction of the right hemiface, followed by paroxysmal respirations and a "painful" facial expression, lasting 15-30 seconds, with no change in consciousness. While the painful facial expression was bilateral and more pronounced in the upper facial muscles, the right hemifacial tonic contraction remained the most prominent change. With one event, the initial semiology was not right hemifacial tonic contraction but presented as paroxysmal respirations with associated simultaneous contractions of respiratory muscles and bilateral upper facial muscles (resulting in blinking), occurring three times in a four second interval, followed by tonic contraction of the right hemiface. During one event, there was definitive lacrimation. Figure 1 shows ictal facial expressions. Semiology of lacrimation, respiratory symptoms, and facial expressions was consistent with crying. Scalp ictal EEG showed onset of left anterior temporal theta range slowing approximately 5-10 seconds after clinical seizure onset. Interictal FDG-PET showed hypometabolism in the left anterior and medial temporal regions. Magnetic resonance imaging was normal.

The patient then underwent intracranial EEG telemetry monitoring, with an eight $\times$ eight contact grid over the left frontal convexity and superior aspect of the temporal lobe, a four contact left subfrontal strip, a four contact medial frontal strip, and two six contact left subtemporal strips. During intracranial EEG telemetry monitoring, initial semiology of all seizures was right hemifacial tonic movements. Figure 2 shows the typical ictal onset and propagation pattern from an intracranial EEG recording. Seizures showed onset from the medial contacts of the posterior subtemporal strip electrodes, which was in the region of the amygdalohippocampal complex, with onset of $20 \mathrm{~Hz}$ activity. Figure 3 shows electrode placement of strip electrodes and grid electrodes which covered the temporal lobe. Correlation of ictal symptomatology with intracranial EEG recordings showed that onset of right hemifacial tonic movements correlated with propagation of beta range ictal discharges to the medial frontal region in all seizures. In referential montages the MF3 electrode shows the highest amplitude activity during initial propagation of the seizure. The delta range activity in the subfrontal electrodes, while correlating with symptoms in the seizure shown in fig 2 was not consistently present at the clinical onset of other seizures recorded during intracranial monitoring.

The patient underwent a left anterior temporal lobectomy with resection of the amygdalo-hippocampal complex, with sparing of the hippocampal body. Post-surgical pathology revealed focal glial nodules and patchy neuronal loss in the amygdala. The patient has been seizure-free for four years postoperatively.

\section{DISCUSSION}

Seizures of medial temporal region onset typically present with an experiential or viscero-sensory aura, followed by impairment of consciousness. ${ }^{1}$ Thus the ictal semiology in this case is atypical. Despite this atypical presentation, multiple tests localised seizures to the left temporal region, and the patient was seizure-free after epilepsy surgery. He

Abbreviation: TLE, temporal lobe epilepsy 

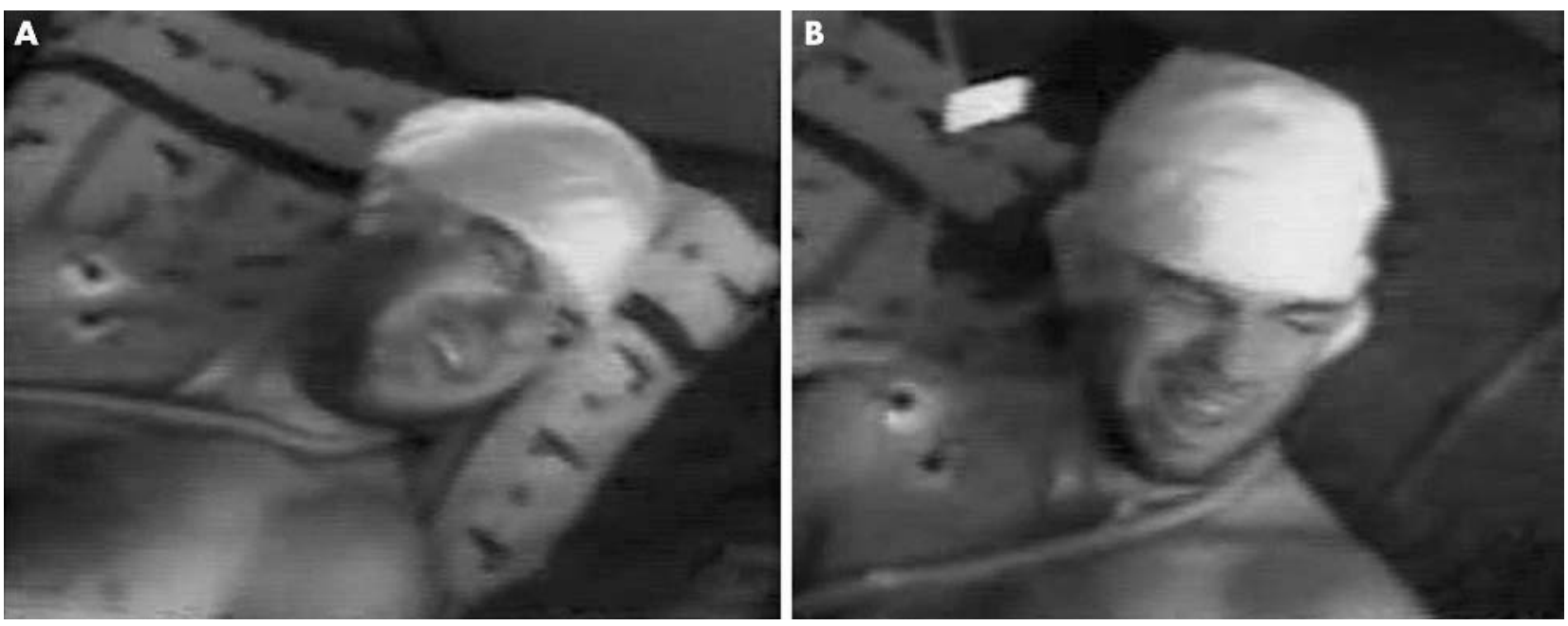

Figure 1 Images of ictal facial movements. (A) The typical tonic right hemifacial movements. The open left eye demonstrates lack of involvement of the left side of the face. (B) A "painful" expression in which both eyes are closed, showing some degree of bilateral facial involvement. Bilateral movements were most pronounced in the upper facial muscles. However, the right hemifacial involvement remains predominant. Written consent for the use of these photographs has been obtained from the patient.

therefore represents a rare instance of seizures of medial temporal region onset presenting with somato-motor symptoms of right hemifacial movements and associated ictal crying.

\section{Somato-motor signs in temporal lobe epilepsy}

Past investigators have observed somato-motor signs in temporal lobe epilepsy (TLE). Bossi et al $l^{3}$ studied 101 seizures from 50 patients during intracranial EEG explorations to define somato-motor manifestations of temporal lobe seizures. They found that somato-motor symptoms occurred "early" (in the first 10 seconds of the ictus) in 10 seizures (9.9\% of their sample). Interestingly, facial involvement was the initial somato-motor involvement in eight of the 10 seizures. Our subject showed "early" somato-motor signs, primarily involving the face. Bossi et al ${ }^{3}$ concluded that ictal somato-motor symptoms in TLE were indicative of spreading of the ictal discharge beyond the temporal lobe. In the context of TLE, past investigators have considered the amygdala as the most likely medial temporal region structure to send projections to the extratemporal structures responsible for associated motor signs, including both dystonic posturing ${ }^{4}$ and somato-motor signs. ${ }^{3}$ However, investigators disagree about the pathways of propagation from the amygdala that causes the motor symptoms. ${ }^{5}$ Gloor reported that there are no direct projections from the amygdala to the motor cortex, and that any behavioural response initiated by amygdaloid activity reflected involvement of the motor system indirectly. ${ }^{6}$ However, recent studies have implied a more direct role of the amygdala in mediating facial motor responses.

There are five cortical motor representations in the nonhuman primate. Five homologous areas have been identified in the human, including the rostral cingulate (M3) and caudal cingulate (M4) regions. ${ }^{7}$ Morecraft et $a l^{8}$ have proposed that M3 and M4 are the regions responsible for "emotional" facial movements. Their studies, in primates, showed bilateral projection from M3 to the upper facial musculature and a contralateral projection from M4 to the lower facial musculature. They also found that the amygdala projects to the somatotopic representations of the cingulate motor field in a highly specific manner. The amygdalocingulate projections provide a basis for anatomicalelectrical-clinical correlations in the present case.
In this case, the seizures began in the medial temporal region but were clinically asymptomatic until they propagated to the cingulate region. For all intracranial ictal EEG recordings, the symptoms of isolated facial movements correlated with onset of beta frequency activity over the medial frontal region, indicating that propagation of the seizures to that region caused the symptoms. We therefore propose that the symptoms in this case were caused by propagation of seizures through amygdalo-cingulate projections, resulting in activation of cortical facial representation regions M3 and M4 in the cingulate. Such a mechanism of symptoms is consistent with the conclusion by Bossi et al that motor symptoms in TLE are related to activation of extratemporal structures. ${ }^{3}$

\section{Ictal crying}

There are relatively few reports of ictal crying. Luciano et al reported seven cases in which ictal or postictal semiology involved crying documented by ictal EEG. ${ }^{9}$ In their series, five patients had a medial temporal onset of seizures, while two had a midline frontal onset. They report subdural electrode monitoring over the cingulate region in one case, which confirmed ictal onset adjacent to a cavernous malformation and the supplementary motor area. A subsequently reported case of post-stroke epilepsy documented repetitive sharp wave discharges, maximal over the right anterior temporal region, during an episode of ictal crying. ${ }^{10}$ Thus past case reports verify ictal EEG onset in the medial temporal and medial frontal regions during seizures associated with ictal crying. The EEG findings in our case are consistent with those of past investigators. However, the correlation of semiology with subdural EEG monitoring in our case documented ictal onset in the medial temporal region, which produces symptoms only after propagation to the cingulate region, providing evidence that temporal lobe onset seizures cause crying by propagating to the cingulate region. In view of the fact, as outlined above, that motor signs are caused by propagation of TLE seizures outside the temporal lobe, propagation to the cingulate provides a plausible explanation for generation of the facial motor components in ictal crying caused by TLE.

\section{Postulations of functional correlates}

Our case involved seizures that always featured right hemifacial tonic contraction, often associated with signs of 


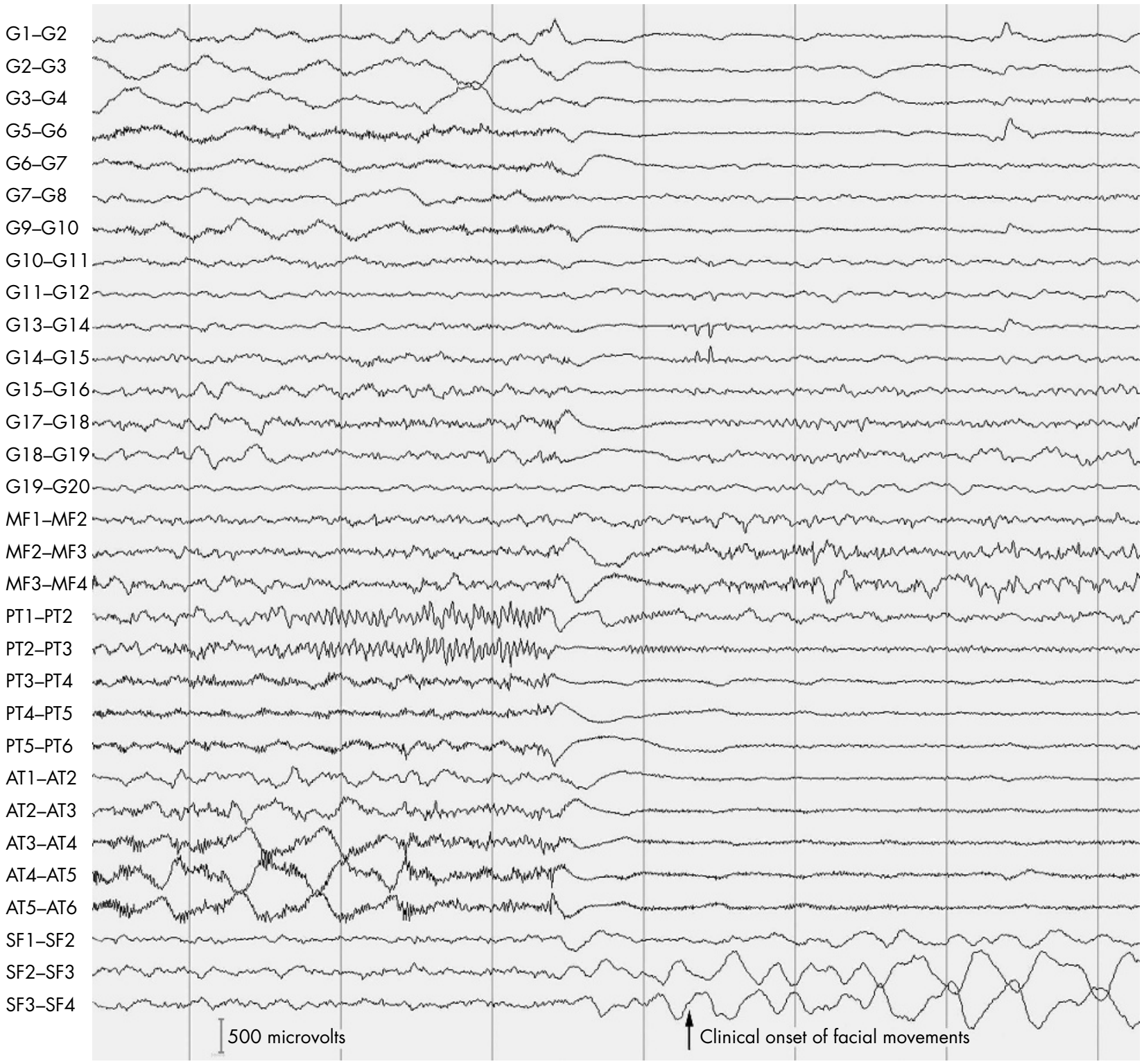

Figure 2 Intracranial ictal EEG recording. Only the grid electrodes covering the temporal lobe are shown in the recording. Electrode abbreviations are as follows: G grid; MF, medial frontal strip; PT, posterior subtemporal strip; AT, anterior subtemporal strip; SF, subfrontal strip. The placement of grid and strip electrodes is shown in fig 3. The arrow at the bottom of the figure shows the time of onset of the right hemifacial movements. Approximately three seconds before the hemifacial movements there was onset of localised $20 \mathrm{~Hz}$ activity over the PT1 and PT2 electrodes, which cover the amygdalo-hippocampal complex. After approximately two seconds, the $20 \mathrm{~Hz}$ activity evolved to cause widespread desynchronisation of all electrodes covering the temporal lobe, after which beta range activity became more prominent over the medial frontal electrodes. The onset of beta range activity in the medial frontal region, which was highest amplitude in electrode 3 on referential recordings, correlated with clinical onset of all seizures.

crying. The case is clinically unique for temporal lobe epilepsy, because the facial movements and signs of crying occurred in isolation from other ictal semiology, suggesting that isolated projections from the amygdala to the cingulate regions were primarily activated during the seizures. The semiology highlights the role of the cingulate in generation of facial movements and crying, and adds further evidence for localisation of "emotional" facial movements ${ }^{8}$ to the cingulate. Because intracranial EEG monitoring of the medial frontal region was a single four contact strip electrode, definitive functional anatomical localisation is limited and indicates maximal activation at the junction of the supplementary motor area and M4 (over electrode MF3). The ictal semiology was not consistent with ictal activation of the supplementary motor area (M2), which typically causes prominent posturing of the upper extremities and associated version of gaze, head, and body. ${ }^{11}$ From a semiological perspective, the seizures correlate best with the proposed functions of M3 and M4. One seizure began with paroxysms of crying, which involved bilateral "eye blinking" movements and paroxysmal respiratory movement. These semiological features are most consistent with predominant activation of M3, which, in classical studies in monkeys, showed respiratory changes and facial movements, ${ }^{12-15}$ and, in more recent studies, bilateral projections for activation of the upper facial musculature. ${ }^{8}$ The semiology of right hemifacial tonic motor movements is most consistent with predominant activation of M4, which shows projections to contralateral lower facial musculature. That the seizures involved the functions of both M3 and M4 suggests that the functions of 

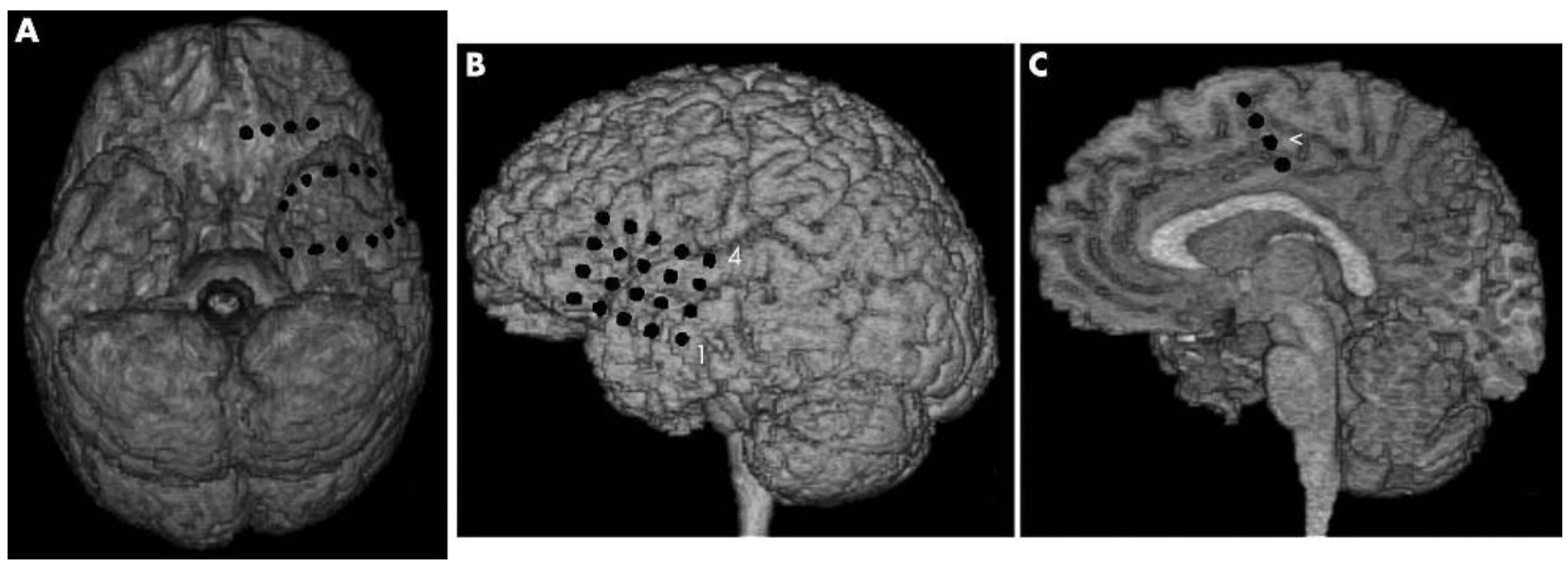

Figure 3 Electrode placement using the computed tomography and magnetic resonance imaging coregistration technique as previously described. ${ }^{2}$ (A) The ventral brain surface showing placement of the subfrontal and subtemporal electrode strips. Electrode 1 is the most medial electrode in all strips in panel A. (B) The left lateral brain surface with the 20 electrodes from the 64 channel grid which cover the lateral temporal lobe. Electrodes 1 and 4 are labelled to orient the viewer to the montage in fig 2. (C) The medial surface of the left hemisphere with the medial frontal electrodes. Electrode 3 is marked with an arrow. The medial frontal strip electrode partially covers both the supplementary motor (M2) and caudal cingulate (M4) areas.

these two facial motor regions are integrated with each other. Integration of function of M3 and M4 is supported by a recent study by Morecraft et al ${ }^{16}$ (case 6), which showed strong interconnections between M3 and M4.

\section{Conclusions}

This case represents a rare example of epileptic seizures of medial temporal onset presenting with semiology of right hemifacial tonic movements and ictal crying. That the patient was successfully treated with epilepsy surgery stresses the clinical importance of associating temporal lobe epilepsy with this semiological presentation. Anatomical-electrical-clinical correlations of this case with cortical regions controlling facial movements are highly suggestive that it represents secondary activation of M3 and M4 (rostral and caudal cingulate motor cortex), giving rise to focal hemifacial movements and ictal crying.

\section{Authors' affiliations}

R E Hogan, V K Rao, Department of Neurology, Saint Louis University School of Medicine, St Louis, Missouri, USA

Competing interests: none declared

Consent was obtained for publication of figure 1

Correspondence to: Dr R Edward Hogan, Department of Neurology, Saint Louis University School of Medicine, 1221 S Grand, Epilepsy Offices, St Louis, MO 63104, USA; hoganr2@slu.edu

Received 5 January 2005

In revised form 18 April 2005

Accepted 26 April 2005

\section{REFERENCES}

1 Palmini A Gloor P. The localizing value of auras in partial seizures: a prospective and retrospective study. Neurology 1992;42:801-8.

2 Hogan RE, Lowe VJ, Bucholz RD. Triple-technique (MR imaging, single-photon emission $\mathrm{CT}$, and CT) coregistration for image-guided surgical evaluation of patients with intractable epilepsy. Am J Neuroradiol 1999;20:1054-8.

3 Bossi L, Munari C, Stoffels C, et al. Somatomotor manifestations in temporal lobe seizures. Epilepsia 1984;25:70-6.

4 Kotagal P, Luders H, Morris HH, et al. Dystonic posturing in temporal lobe seizures. Neurology 1989:39:1271.

5 Bennett DA, Ristanovic RK, Morrell F, et al. Dystonic posturing in temporal lobe seizures. Neurology 1989;39:1270-1.

6 Gloor P. The amygdaloid system. In: The temporal lobe and limbic system. New York: Oxford University Press, 1997:591-721.

7 Morecraft RJ, Stilwell-Morecraft KS, Rossing WR. The motor cortex and facial expression: new insights from neuroscience. Neurologist 2004; 10:235-49.

8 Morecraft RJ, Lovie JL, Herrick JL, et al. Cortical innervation of the facial nucleus in the non-human primate: a new interpretation of the effects of stroke and related subtotal brain trauma on the muscles of facial expression. Brain 2001;124:176-208

9 Luciano D, Devinsky O, Perrine K. Crying seizures. Neurology 1993:43:2113-17.

10 Wang DZ, Steg RE, Futrell N. Crying seizures after cerebral infarction. I Neurol Neurosurg Psychiatry 1995;58:380-1.

11 Luders HO, Noachtar S. Classification of epileptic syndromes and paroxysmal events. In: Atlas of epileptic seizures and syndromes. Philadelphia: WB Saunders, 2001:46-52.

12 Kaada BR, Pribram KH, Epstein JA. Respiratory and vascular responses in monkeys from temporal pole, insula, orbital surface and cingulate gyrus. J Neurophysiol 1949;12:348-56.

13 Showers MJC, Crosby EC. Somatic and visceral responses from the cingulate gyrus. Neurology 1958;8:561-5.

14 Showers MJC. The cingulate gyrus: additional motor area and cortical autonomic regulator. J Comp Neurology 1959;1 12:231-87.

15 Ward AA. The cingulate gyrus: area 24. J Neurophysiol 1948;11:13-23.

16 Morecraft RJ, Cipolloni PB, Stilwell-Morecraft KS, et al. Cytoarchitecture and cortical connections of the posterior cingulate and adjacent somatosensory fields in the rhesus monkey. J Comp Neurol 2004;469:37-69. 